\title{
No Free Lunch for Noise Prediction
}

\author{
Malik Magdon-Ismail \\ California Institute of Technology, Pasadena, CA 91125, U.S.A.
}

No-free-lunch theorems have shown that learning algorithms cannot be universally good. We show that no free funch exists for noise prediction as well. We show that when the noise is additive and the prior over target functions is uniform, a prior on the noise distribution cannot be updated, in the Bayesian sense, from any finite data set. We emphasize the importance of a prior over the target function in order to justify superior performance for learning systems.

\section{Introduction}

Already established in the learning theory literature are a set of "no-freelunch" (NFL) theorems for various types of learning systems (Wolpert, 1996a, 1996b; Zhu, 1996; Cataltepe, Abu-Mostafa, \& Magdon-Ismail, 1999) and for optimization methods (Wolpert \& Macready, 1997). The essential content of these theorems is that no learning algorithm can be universally good. A learning algorithm that performs exceptionally well in certain situations will perform comparably poorly in other situations. Thus, in particular, random algorithms that always yield average performance, cross validation, bootstrapping, and anti-cross validation are all, in some sense, on an equal footing. Stopping early at a higher training error than the minimum achievable gains nothing if the hypotheses yielding that training error are chosen with equal probability. A search technique that performs well on one cost function will perform poorly on another cost function. These existing results demonstrate that for certain problems, given a certain criterion of performance, it is not possible to do well consistently in the most general setting. Thus, one needs to make some assumptions about the underlying structure of the problem to be able to claim any kind of superior performance.

Here we look at the problem of inferring the noise distribution from a data set. The existing NFL results look at a problem of learning a dependency (not necessarily deterministic) from a finite data set and making statements about the out-of-sample performance. The problem we treat here is to deduce properties of the noise distribution (for example, the noise variance) from a finite data set that consists of input-output pairs. The outputs have a deterministic dependence on the inputs (the target function) and a noisy component. One approaches the problem with some prior belief about the noise distribution, and one hopes that the data will enable a fine-tuning of that prior 


\section{Which data set has more noise?}

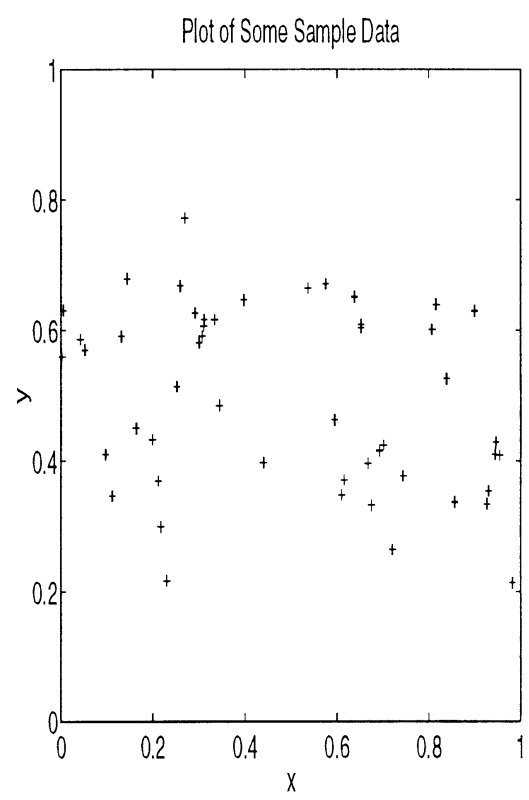

(a)

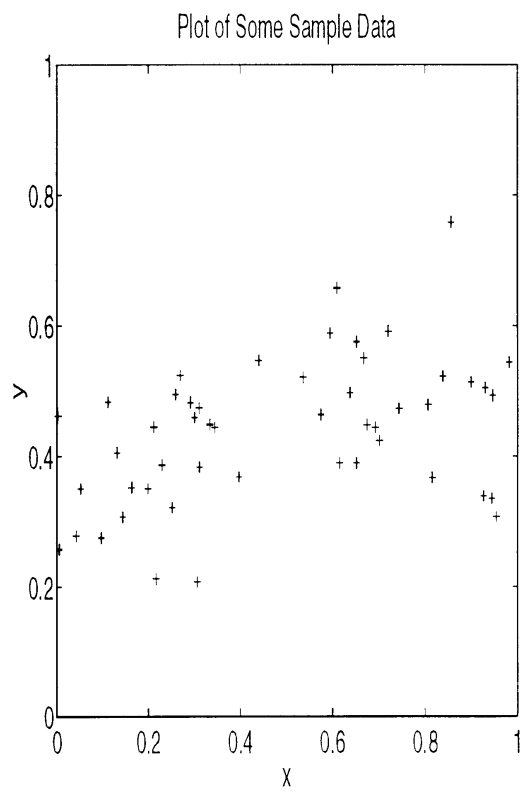

(b)

Figure 1: Two sample noisy data sets. The sets were created by taking two functions and adding noise to them. The $x$-values are the same in both cases.

belief to the extent that more precise statements can be made about the properties of the noise. An accurate estimate of the noise distribution is useful because the noise sets a fundamental limit on the performance of a learning system (Cortes, Jackel, \& Chiang, 1994). Noise may play other roles as well: for example, in financial markets, the noise level is itself a tradable quantity.

Our criterion for selection of a noise distribution will be to maximize its posterior probability. We will show that having a uniform prior on the possible realizations of the target function leads to no update for a wellbehaved prior on the noise distributions (in the Bayesian sense) from any finite data set. Thus the posterior over noise distributions will equal the prior, and we may as well have not even looked at the data. One can compare this to the analogous NFL result of Wolpert: if the prior over target functions is uniform, then the out-of-sample error is constant, and thus one need not have even looked at the data before picking a hypothesis function. Let us consider the question posed in Figure 1. Which data set has more noise? Figure 2 shows the same data, but this time with the function that created 


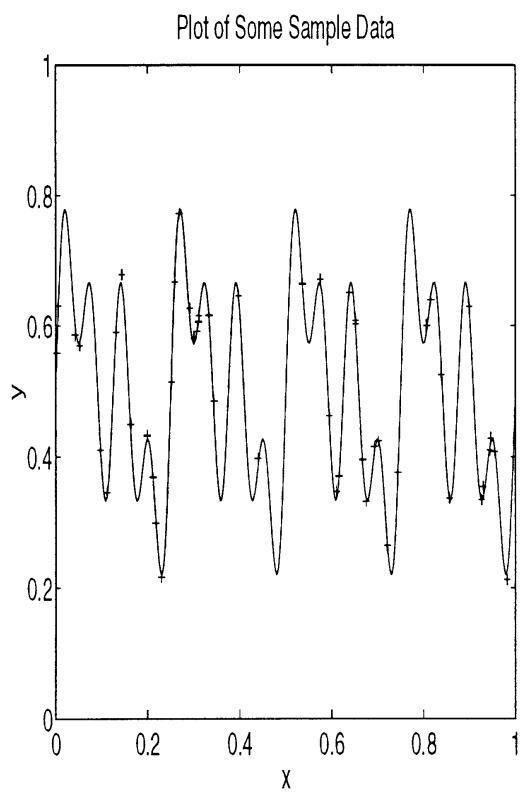

(a)

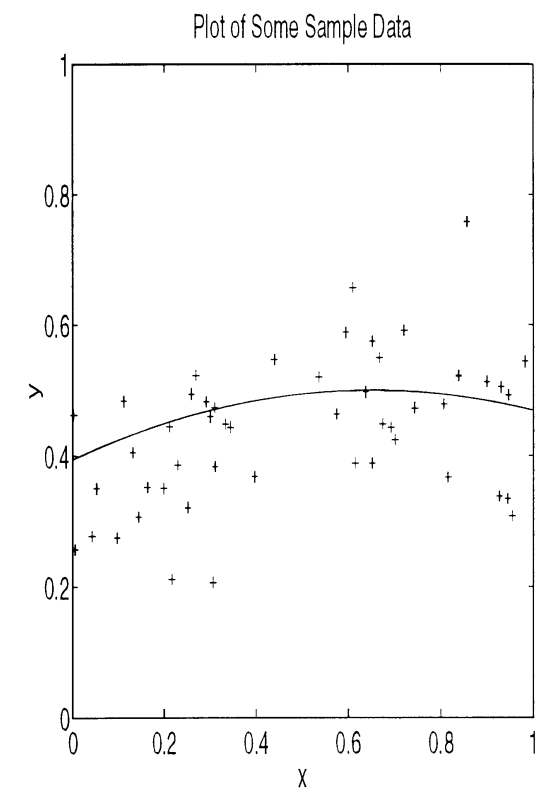

(b)

Figure 2: Noisy data and the functions that generated them. The same noisy data sets that were shown in Figure 1 and the functions that were used to produce them. In (b), the noise variance is roughly six times larger.

the data. Now which data set has more noise? The task is considerably easier given this extra information. We will show that some extra information of this form is essential.

This article is organized as follows. We start with an example in section 2, where we attempt to predict a noise variance when learning using linear models. In this section we show that one method for predicting the noise variance using a bootstrap technique does not work in the naive form presented. As a result, one might therefore spend a lot of effort trying to find a more sophisticated technique that might work. That this would be fruitless (for this specific case and the more general case) is the purpose of the remainder of the article. In section 3 , we set up the problem of noise prediction in general; section 4 presents the main results, beginning with the simple case of boolean functions and continuing to the more general case. We show that information about the noise distribution cannot be obtained from a finite data set if the target distribution is assumed uniform. We conclude with section 5, where we also discuss the implications of the NFL theorems. 


\section{An Example Using Linear Models}

In this section we illustrate the conclusions of this chapter for the case of linear learning models on the input space $\mathbf{R}^{d}$. It is not our goal to be strictly rigorous, but rather to illustrate the point. Let the hypothesis functions be of the form

$$
g_{\mathbf{w}}(\mathbf{x})=\mathbf{w} \cdot \mathbf{x}+\mathbf{w}_{0},
$$

where $\mathbf{w}, \mathbf{w}_{0}$ are called the weights. We are given a data set $\mathcal{D}=\left\{\mathbf{x}_{\alpha}, y_{\alpha}=\right.$ $\left.f\left(\mathbf{x}_{\alpha}\right)+n_{\alpha}\right\}_{\alpha=1}^{N} \cdot f(\cdot)$ is some deterministic function (we place no other restrictions on the function) and $n_{\alpha}$ is noise, drawn independently and identically distributed with zero mean and variance $\sigma^{2}$. We use mean squared deviation as the measure of error. Define the training error as

$$
\mathcal{E}_{t r}=\frac{1}{N} \sum_{\alpha}\left(\mathbf{w} \cdot \mathbf{x}_{\alpha}+w_{0}-y_{\alpha}\right)^{2}
$$

Define $\mathbf{w}^{*}, \mathbf{w}_{0}^{*}$ as those weights that minimize $\mathcal{E}_{t r}$. It can then be shown that at this minimum, the expected training error is given by (theorem 4 in the appendix $)^{1}$

$$
\left\langle\mathcal{E}_{t r}^{*}\right\rangle_{\mathcal{D}, \mathbf{n}}=E_{0}+\sigma^{2}-\frac{\sigma^{2}(d+1)+B}{N}+O\left(\frac{1}{N^{\frac{3}{2}}}\right) .
$$

We use $\langle\cdot\rangle$ to denote expectations, and the expectation here is over the possible data sets (the input space and the noise, where we have made explicit the expectation over the noise). ${ }^{2} E_{0}$ is the bias, where the bias is the expected training error of the best hypothesis function in our learning model. $B$ is a constant given by theorem 4 . For the purposes of this section, the exact expression for $B$ is not essential. It suffices to know that $B$ is related to the variance of a sample statistic around its population value.

The goal here is to estimate the noise variance, $\sigma^{2}$. The training error used as an estimate of the noise variance is asymptotically biased. For large $N$, it overpredicts the noise variance by the bias, $E_{0}$. Since we know the form of $\left\langle\mathcal{E}_{t r}^{*}\right\rangle$, perhaps we can do better. We can sample $N_{1}$ data points from the $N$

\footnotetext{
${ }^{1}$ We use standard order notation:

$$
\begin{aligned}
& f(N)=o(g(N)) \Rightarrow f(N) / g(N) \stackrel{N \rightarrow \infty}{\longrightarrow} 0 \\
& f(N)=O(g(N)) \Rightarrow f(N) \leq C g(N) \text { for some } C>0 .
\end{aligned}
$$
}

\footnotetext{
${ }^{2}$ In the language of the NFL theorems (Wolpert, 1996b), $\left\langle\mathcal{E}_{t r}^{*}\right\rangle_{\mathcal{D}, \mathbf{n}}$ is $E(C \mid f, m)$ where the on-training-set likelihood and the quadratic loss function are used.
} 
data points and estimate the training error. We use bootstrapping (Shao \& $\mathrm{Tu}, 1996)$ to estimate $\left\langle\mathcal{E}_{t r}^{*}\left(N_{1}\right)\right\rangle$, and by varying $N_{1}$, we can fit the resulting dependence of $\left\langle\mathcal{E}_{t r}^{*}\left(N_{1}\right)\right\rangle$ on $1 / N_{1}$ using equation 2.3 . Let the slope of this fit be $\hat{A}$. From equation 2.3 , we see that $\hat{A}$ is an estimate for $\sigma^{2}(d+1)+B$; therefore, $\hat{A} /(d+1)$ used as an estimate of $\sigma^{2}$ is off by $B /(d+1)$. Perhaps we can estimate $B$ by the bootstrap as well. Given a bootstrapped estimate for $B$, we can combine it with $\hat{A}$ to obtain an estimate for $\sigma^{2}$. A more careful analysis presented in the appendix shows that this too leads to failure.

Thus, to get information on $\sigma^{2}$, perhaps a more subtle approach such as looking at terms with higher order in $1 / N$ would work. Perhaps some method other than simply using the training error would yield a better result. Instead of using linear models, why not use a more complex model for which $E_{0}$ is zero? Then at least asymptotically, the training error will approach $\sigma^{2}$ (provided that the model is not too complex). However, for any finite $N$, we run the risk of overfitting the data, perhaps even obtaining an estimate $\sigma^{2}=0$. Perhaps there is a systematic way of choosing the model complexity as a function of $N$ to get an optimal noise variance estimate.

The next few sections show that such an exhaustive search will necessarily be fruitless unless we make some statement about $f$. In this case it would suffice to tell us "how good our model is at estimating $f^{\prime \prime}$ (i.e., $E_{0}$ ). Rather than explore every other potential method for estimating $\sigma^{2}$ in this linear scenario, we will set up a more general framework for the estimation of noise distributions and show that unless some assumptions are made about the target function, all noise models are just as probable, given the data. The intuition is that the data points are consistent with any noise level if we do not restrict the target function. We pursue these issues next.

\section{Problem Set-Up}

A finite data set $\mathcal{D}=\left\{\mathbf{x}_{\alpha}, y_{\alpha}\right\}_{\alpha=1}^{l}$ is given, where $\mathbf{x}_{\alpha} \in \mathbf{R}^{d}, y_{\alpha}=f\left(x_{\alpha}\right)+n_{\alpha}$, $f: \mathbf{R}^{d} \rightarrow \mathbf{R}$ and the data set consists of $l$ data points. ${ }^{3}$ We concentrate on the case where the noise distribution is fixed. The results can be modified to accommodate noise distributions that vary with $\mathbf{x}$. It will always be understood that $\alpha$ indexes points in the data set.

We concentrate on compact spaces and assume that the input space is the unit hypercube in $d$ dimensions. Let the output space be the bounded interval $[-C, C]$. Discretize the input and output spaces as follows. Let the input variable, $\mathbf{x}$, take on values in the set

$$
\mathcal{X}=\left\{0, \Delta_{x}, 2 \Delta_{x}, \ldots, 1-\Delta_{x}, 1\right\}^{d}
$$

\footnotetext{
${ }^{3}$ We use $l$ rather than the customary $N$ to avoid confusions with $N_{x}, N_{y}$, to be defined shortly.
} 


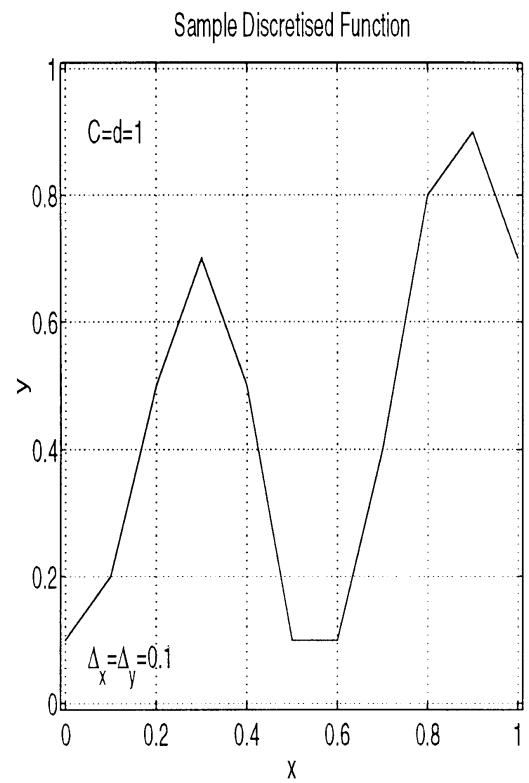

(a)

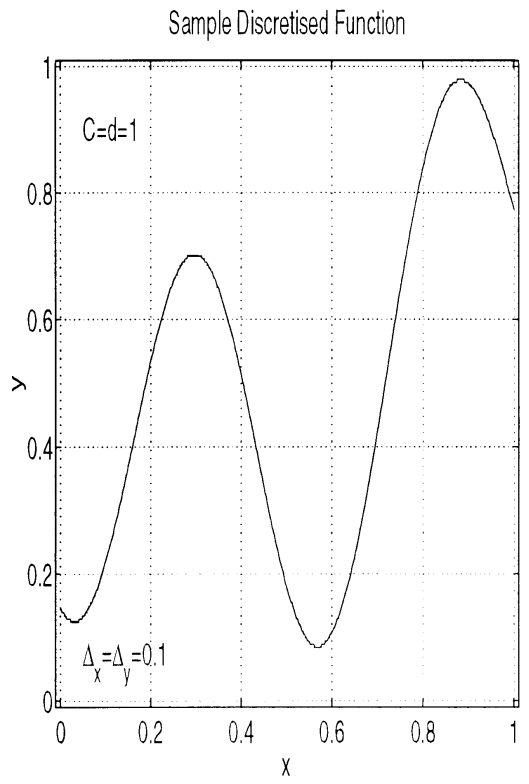

(b)

Figure 3: Discretized function space. The space has only a finite number of functions. An arbitrary precision can be obtained by making the grid finer and finer.

so there are $N_{x}=1+\frac{1}{\Delta_{x}}$ possible values for each input variable. Let the output variable, $y$, take a value in the set

$$
\mathcal{Y}=\left\{-C,-C+\Delta_{y}, \ldots, 0, \Delta_{y}, 2 \Delta_{y}, \ldots, C-\Delta_{y}, C\right\} .
$$

There are $N_{y}=\frac{2 C}{\Delta_{y}}+1$ possible $y$ values. A function $(f)$ will be a mapping, $f: \mathcal{X} \rightarrow \mathcal{Y}$. There are $\Gamma=N_{y}{ }^{{ }^{d}{ }^{d}}$ different functions. By making $\Delta_{x}, \Delta_{y}$ as small as we choose, we can achieve an arbitrary precision (for example, see Figure 3). In all applications, only a finite number of functions are available (computers are finite precision machines). Due to this finiteness, we can define a probability distribution on the function space-a prior over the function space. One natural prior is to take this distribution to be uniform. Therefore to every function we assign a probability of $\frac{1}{\Gamma}$.

We assume that $C$ can be made as large as we wish. Further, without loss of generality, we can scale the outputs by $\frac{1}{\Delta_{y}}$; therefore we can assume that $y \in\{-\tilde{C},-\tilde{C}+1,-\tilde{C}+2, \ldots, \tilde{C}-1, \tilde{C}\}, N_{y}=2 \tilde{C}+1$ and $\tilde{C}=C / \Delta_{y}$. With this representation, the noise $n \in\{0, \pm 1, \pm 2, \ldots\}$ has a distribution given by 
a vector $\mathbf{P}$, where $P_{i}=\operatorname{Pr}[n=i]$ and $\sum_{i} P_{i}=1$. Assume a prior probability measure on the possible noise distributions $g(\mathbf{P})$. We are interested in the Bayesian posterior on these noise distributions,

$$
g(\mathbf{P} \mid D)=\frac{\operatorname{Pr}[D \mid \mathbf{P}] g(\mathbf{P})}{\operatorname{Pr}[\mathcal{D}]},
$$

where $\left.\operatorname{Pr}[\mathcal{D}]=\sum_{\mathbf{P}} \operatorname{Pr}[\mathcal{D} \mid \mathbf{P}]\right\}(\mathbf{P})$. We can calculate $\operatorname{Pr}[D \mid \mathbf{P}]$ as follows:

$$
\operatorname{Pr}[\mathcal{D} \mid \mathbf{P}, f]=\prod_{\alpha=1}^{l} \operatorname{Pr}\left[n_{\alpha}=y_{\alpha}-f\left(\mathbf{x}_{\alpha}\right)\right]
$$

Note that the right-hand side implicitly depends on $\mathbf{P}$ through the probabilities for the noise realizations $n_{\alpha}$. Multiplying by $P[f]$ and summing over $f$, we have

$$
\begin{aligned}
\operatorname{Pr}[D \mid \mathbf{P}] & =\frac{1}{\Gamma} \sum_{f\left(\mathbf{x}_{1}\right)} \sum_{f\left(\mathbf{x}_{2}\right)} \cdots \sum_{f\left(\mathbf{x}_{N_{x}}\right)} \prod_{\alpha=1}^{l} \operatorname{Pr}\left[n_{\alpha} \mid \mathbf{f}, \mathcal{D}\right] \\
& \stackrel{(a)}{=} \frac{1}{N_{y}^{l}} \prod_{\alpha=1}^{l} \sum_{f\left(x_{\alpha}\right)=-C}^{C} \operatorname{Pr}\left[n_{\alpha} \mid \mathbf{f}, \mathcal{D}\right],
\end{aligned}
$$

where we have used the notation $\operatorname{Pr}\left[n_{\alpha} \mid \mathbf{f}, \mathcal{D}\right]$ to mean $\operatorname{Pr}\left[n_{\alpha}=y_{\alpha}-f\left(\mathbf{x}_{\alpha}\right)\right]$; (a) follows when we sum over all points not in the data set, and we have used the fact that $\operatorname{Pr}[f]=1 / \Gamma-$ uniform prior.

\section{No Free Lunch for Noise Prediction}

4.1 Boolean Functions. We use the following notation:

$$
\begin{aligned}
\mathbf{x} & \in\{0,1\}^{d} \\
f: \mathbf{x} & \rightarrow\{0,1\} \\
(1-p) \in[0,1] & =\text { probability of flip. }
\end{aligned}
$$

There are $\Gamma=2^{2^{d}}$ possible functions.

Theorem 1. Let $g(p)$ be the prior distribution for the noise parameter, $p$. If the prior distribution on the possible functions is uniform then $g(p \mid \mathcal{D})=g(p)$.

Proof. Let $m_{i}$ be the number of times $f_{i}$ agrees with $\mathcal{D}$ where we use $i$ to index functions. Then $\operatorname{Pr}\left[\mathcal{D} \mid p, f_{i}\right]=p^{m_{i}}(1-p)^{l-m_{i}}$, so

$$
\operatorname{Pr}\left[\mathcal{D}, f_{i} \mid p\right]=\operatorname{Pr}\left[\mathcal{D} \mid p, f_{i}\right] \operatorname{Pr}\left[f_{i} \mid p\right]=\frac{1}{\Gamma} p^{m_{i}}(1-p)^{l-m_{i}}
$$


Let $\rho\left(m_{i}\right)$ be the number of functions agreeing exactly $m_{i}$ times with the data set D. $\rho\left(m_{i}\right)=\left(\begin{array}{c}l \\ m_{i}\end{array}\right) 2^{2^{d}-l}=\left(\begin{array}{c}l \\ m_{i}\end{array}\right) 2^{-l} \Gamma$. Summing equation 4 .1 over $f_{i}$, we get

$$
\begin{aligned}
\operatorname{Pr}[\mathcal{D} \mid p] & =\sum_{f_{i}} \operatorname{Pr}\left[\mathcal{D}, f_{i} \mid p\right] \\
& =\frac{1}{\Gamma} \sum_{m_{i}=0}^{l} p^{m_{i}}(1-p)^{l-m_{i}} \rho\left(m_{i}\right) \\
& =\left(\begin{array}{cc}
\frac{1}{2^{l}} \sum_{m_{i}=0}^{l} p^{m_{i}}(1-p)^{l-m_{i}} \\
l m_{i}
\end{array}\right) \\
& =2^{-l} \quad
\end{aligned}
$$

independent of $p$. Therefore,

$$
g(p \mid \mathcal{D})=\frac{\operatorname{Pr}(\mathcal{D} \mid p) g(p)}{\operatorname{Pr}(\mathcal{D})}=g(p) .
$$

4.2 Extension to More General Functions. We would like to extend the previous analysis to the case where the output is not binary and the input is the discretized version of $[0,1]^{d}$. Figure 4 illustrates the conclusions of this section on a particular discretized grid. This section is somewhat technical. Its essential content is contained in theorem 3 , which formalizes the intuition that if every target function value is equally likely, then each noise realization for every data point is equally likely. Hence nothing can be deduced about the relative likelihoods of different noise realizations.

4.2.1 Cyclic Noise. When the noise is additive modulo $N_{y}$, then $\mathbf{P}=$ $\left[P_{0}, P_{1}, \ldots, P_{N_{y}-1}\right]$ fully specifies the noise distribution.

Example. The binary case with probability of flip $1-p$ we have $N_{y}=2$, $P_{0}=p, P_{1}=1-p$

Lemma 1. $\sum_{f\left(x_{\alpha}\right)} \operatorname{Pr}\left[n_{\alpha} \mid \mathbf{f}, \mathcal{D}\right]=1$ for all $\mathbf{x}_{\alpha} \in \mathcal{D}$.

Proof.

$$
\sum_{f\left(x_{\alpha}\right)=-C}^{C} \operatorname{Pr}\left[n_{\alpha} \mid \mathbf{f}, \mathcal{D}\right]=\sum_{n_{\alpha}=y_{\alpha}+C}^{y_{\alpha}-C} \operatorname{Pr}\left[n_{\alpha} \mid \mathbf{f}, \mathcal{D}\right]=\sum_{n_{\alpha}=0}^{N_{y}-1} P_{n_{\alpha}}=1
$$

Combining lemma 1 with equation 3.5, we have the following theorem:

Theorem 2. Let $g(\mathbf{P})$ be the prior distribution for the cyclic noise. If the prior distribution for the functions is uniform, then $g(\mathbf{P} \mid D)=g(\mathbf{P})$. 


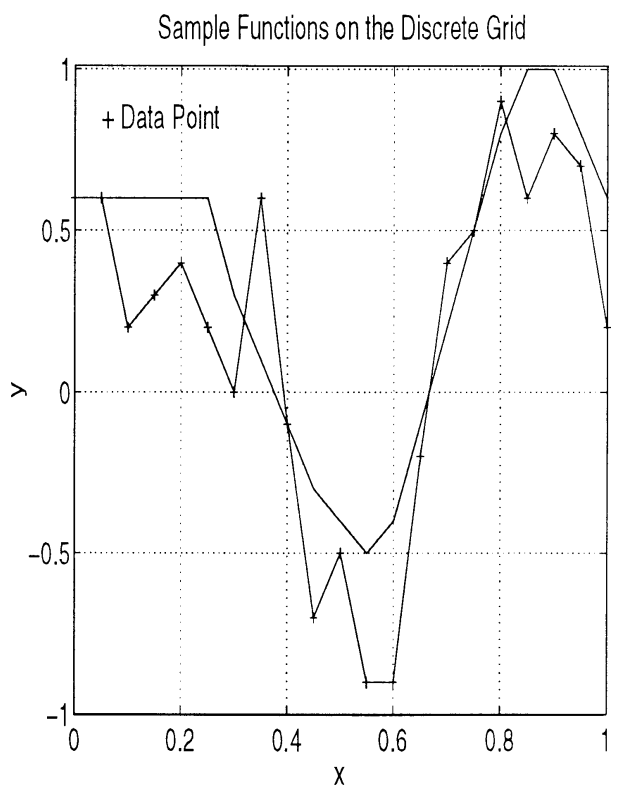

Figure 4: Illustration of no noisy free lunch (NNFL). Suppose that the two functions shown are both equally likely (probability 1/2) to be the function that created the data. Then the two hypotheses, noise $=0$ and noise $>0$, are still equally likely to be true if they were a priori equally likely to be true.

Proof. By lemma 1 and equation 3.5, $\operatorname{Pr}[D \mid \mathbf{P}]=\frac{1}{N_{y}{ }^{l}}$. Therefore,

$$
g(\mathbf{P} \mid D)=\frac{\operatorname{Pr}[D \mid \mathbf{P}] g(\mathbf{P})}{\operatorname{Pr}[\mathcal{D}]}=g(\mathbf{P}) .
$$

Note that the boolean case is a special case of the cyclic noise case. In the language of information theory (Cover \& Thomas, 1991), the noise forms a symmetric channel between the function value and the output value. A uniform distribution for the function values induces a uniform distribution on the output values, independent of the details of the symmetric noisy channel. Hence, observing the output conveys no information about the noisy channel. This is essentially the content of theorem 2.

4.2.2 Thresholded Additive Noise. Noise is added to the target, and then the resultant value is thresholded, so,

$$
y_{i}=\left\{\begin{array}{l}
\min \left\{C, f\left(\mathbf{x}_{i}\right)+n_{i}\right\}, f\left(\mathbf{x}_{i}\right)+n_{i} \geq 0 \\
\max \left\{-C, f\left(\mathbf{x}_{i}\right)+n_{i}\right\}, f\left(\mathbf{x}_{i}\right)+n_{i}<0
\end{array}\right.
$$


$\mathbf{P}=\left[P_{0}, P_{ \pm 1}, P_{ \pm 2}, \ldots\right]$. Unlike the previous case, edge effects make $\operatorname{Pr}[D \mid \mathbf{P}]$ dependent on $\mathbf{P}$. These effects can be made as small as we please by allowing $C$ to be as large as we please. The intuition is that the data set is finite $\left(y_{i}\right.$ is bounded), and so, because the noise distribution must decay to zero, if $C$ is large enough, the probability that $f\left(x_{\alpha}\right)$ is close to the edge and $y_{\alpha}$ so far away becomes negligible. This is formalized in the next lemma.

Lemma 2. Given $\epsilon>0, \exists C^{*}$ such that for all $C \geq C^{*}$,

$$
\frac{1}{N_{y}{ }^{l}}(1-\epsilon) \leq \operatorname{Pr}[D \mid \mathbf{P}] \leq \frac{1}{N_{y}{ }^{l}} .
$$

$\left(N_{y}=2 C+1\right.$ and intuitively speaking, $\left.\epsilon \stackrel{C \rightarrow \infty}{\longrightarrow} 0\right)$.

Proof. Because $\left\{P_{i}\right\}$ is summable, there exists $\eta$ such that $\sum_{|i|>\eta} P_{i}<\frac{\epsilon}{l}$. Let $y_{\max }=\max \left\{y_{i}\right\}$ and $y_{\min }=\min \left\{y_{i}\right\}$. Choose $C^{*}>\max \left\{\left|y_{\max }+\eta\right|,\left|y_{\min }-\eta\right|\right\}$ and let $C \geq C^{*}$. We then have

$$
\begin{aligned}
\sum_{f\left(x_{\alpha}\right)=-C}^{C} \operatorname{Pr}\left[n_{\alpha} \mid \mathbf{f}, \mathcal{D}\right] & =\sum_{n_{\alpha}=y_{\alpha}-C}^{y_{\alpha}+C} \operatorname{Pr}\left[n_{\alpha} \mid \mathbf{f}, \mathcal{D}\right] \\
& =1-\sum_{n_{\alpha}<y_{\alpha}-C} \operatorname{Pr}\left[n_{\alpha} \mid \mathbf{f}, \mathcal{D}\right]-\sum_{n_{\alpha}>y_{\alpha}+C} \operatorname{Pr}\left[n_{\alpha} \mid \mathbf{f}, \mathcal{D}\right] \\
& \geq 1-\frac{\epsilon}{l},
\end{aligned}
$$

where the last inequality follows because by construction $y_{\max }-C<-\eta$ and $y_{\min }+C>\eta$. Using equation 3.5 , we have

$$
\frac{1}{N_{y}{ }^{l}} \geq \operatorname{Pr}[D \mid \mathbf{P}] \geq \frac{1}{N_{y}{ }^{l}}\left(1-\frac{\epsilon}{l}\right)^{l} .
$$

The lemma now follows by using the inequality $(1-x)^{n} \geq 1-n x$ for $0 \leq x \leq 1$.

Thus, we see that by choosing $C$, the bound on our function $f$ to be large enough, the noise distribution has almost no effect on the probability of obtaining a particular data set. All data sets become equally probable, in the limit of large $C$. This tells us that the data should not be telling us any more about the noise distribution than we already know. The prior distribution for the noise is unaffected given any finite data set. To formalize this intuition, we need to impose a technical condition on the prior over the noise distribution space. 
Definition 1. Let $0<\epsilon \leq 1$. Define $\eta_{\mathbf{P}}(\epsilon)$ by

$$
\eta_{\mathbf{P}}(\epsilon)=\min \left\{\eta: \sum_{|i|>\eta} P_{i}<\epsilon\right\} .
$$

For all $0<\epsilon \leq 1, \eta_{\mathbf{P}}(\epsilon)<\infty$, by the summability of $\mathbf{P}$.

Definition 2. Let $0<\epsilon \leq 1$. Define $\bar{\eta}(\epsilon)$ by

$$
\bar{\eta}(\epsilon)=\sup _{\mathbf{P}}\left\{\eta_{\mathbf{P}}(\epsilon)\right\}
$$

where the sup is taken over $\mathbf{P} \in \operatorname{support}\{g(\mathbf{P})\}$.

Definition 3. We say that a prior on the space in which $\mathbf{P}$ is defined, $g(\mathbf{P})$, is totally bounded if

$$
\bar{\eta}(\epsilon)<\infty
$$

for all $\epsilon>0$.

Essentially this means that the support set for the prior on the noise distributions is not "too large." For example, a prior over the noise distribution space that has support over only a finite number of points (i.e., that is nonzero only for a finite number of noise distributions) is totally bounded. We are now in a position to make precise the intuition mentioned above.

Theorem 3 (No Noisy Free Lunch (NNFL)). Let the prior over noise distributions, $g(\mathbf{P})$, be totally bounded. For all $0<\epsilon<1, \exists C^{*}>0$ such that for all $C \geq C^{*}$,

$$
(1-\epsilon) g(\mathbf{P}) \leq g(\mathbf{P} \mid D) \leq \frac{g(\mathbf{P})}{1-\epsilon}
$$

Therefore,

$$
\lim _{C \rightarrow \infty} g(\mathbf{P} \mid D)=g(\mathbf{P})
$$

because $\epsilon \rightarrow 0$ can be attained by letting $C \rightarrow \infty$.

Proof. Following lemma 2, now let $C^{*}>\max \left\{\left|y_{\max }+\bar{\eta}(\epsilon)\right|,\left|y_{\min }-\bar{\eta}(\epsilon)\right|\right\}$ and let $C \geq C^{*}$. Then lemma 1 applies uniformly to every $\mathbf{P} \in \operatorname{support}\{g(\mathbf{P})\}$. 
Therefore equation 4.2 holds for every $\mathbf{P} \in \operatorname{support}\{g(\mathbf{P})\}$.

$$
\begin{aligned}
g(\mathbf{P} \mid D) & =\frac{\operatorname{Pr}[D \mid \mathbf{P}] g(\mathbf{P})}{\operatorname{Pr}[\mathcal{D}]} \\
& =\frac{\operatorname{Pr}[D \mid \mathbf{P}] g(\mathbf{P})}{\sum_{\mathbf{P}} \operatorname{Pr}[D \mid \mathbf{P}] g(\mathbf{P})} .
\end{aligned}
$$

Using equation 4.2, an upper bound can be obtained by substituting $\operatorname{Pr}[D \mid \mathbf{P}]$ with $1 / N_{y}{ }^{l}$ in the numerator and $(1-\epsilon) / N_{y}{ }^{l}$ in the denominator. To get a lower bound, we do the opposite. Then, using the fact that $\sum_{\mathbf{P}} g(\mathbf{P})=1$, we get equation 4.3 .

Therefore, having no bound on $f$ and allowing all $f$ 's to be equally likely does not allow anything new to be deduced about the noise distribution given any finite data set. It should also be clear how to extend these results to the case where the noise distribution has $\mathbf{x}$ dependence. One looks at each point independently.

The nonthresholded additive noise model is asymptotically obtained by letting $C$ become arbitrarily large. Further, the results apply to arbitrary $\Delta_{\mathbf{x}}, \Delta_{y}$, so by allowing $\Delta_{\mathbf{x}}, \Delta_{y} \rightarrow 0$, these results can be applied to the nondiscretized model in this asymptotic sense.

\section{Discussion}

The posterior $g(\mathbf{P} \mid D)$ is given by

$$
g(\mathbf{P} \mid D) \propto g(\mathbf{P}) \sum_{f} \operatorname{Pr}[\mathcal{D} \mid \mathbf{P}, f] P[f],
$$

where $P[f]$ is the prior over target functions. We have demonstrated that when $P[f]$ is "uniform," it is not possible to update a prior on the noise distribution from a finite data set, provided that a certain technical condition is satisfied. A nontrivial $P[f]$ is needed for $g(\mathbf{P} \mid D)$ to be different from $g(\mathbf{P})$. Exactly how $P[f]$ will factor into $g(\mathbf{P} \mid D)$ is given by equation 5.1 .

The result is more useful than it appears at first sight, for one might argue that no one ever has a uniform prior. A restatement of the result is: Given two noise distributions, $\mathbf{P}_{1}$ and $\mathbf{P}_{2}$, there are just as many (nonuniform) priors that favor $\mathbf{P}_{1}$ over $\mathbf{P}_{2}$ (when one weights by the amount by which $\mathbf{P}_{1}$ is favored) as vice versa.

This result fits into a set of NFL theorems that might be considered a collection of negative results, offering no hope for learning theory. The situation is considerably more positive, however. One is never in a situation with a uniform $P[f]$. Further, it is rare that no information about $P[f]$ is known (excepting that it is nonuniform). Therefore, incorporating $P[f]$ into 
the noise prediction or learning algorithm is necessary in order to claim superior performance. Distribution-independent bounds such as the VC bounds (Vapnik, 1995) say that with high probability, the training error is close to the test error given some fixed target function $f$. But VC results do not guarantee that the training error will be low with high probability; therefore they are of little practical use (for a deeper discussion of the subtleties underlying the connection between NFL and VC theory, see Wolpert $(1995,1996 b) .{ }^{4}$ How could one guarantee that a training error close to zero is achievable? One has to use the prior information available on the target function. Along a similar vein, results that exemplify the superiority of algorithm A over algorithm B on a certain benchmark problem are also of little use, for the reverse will be true on some other "benchmark" problem, and we never know which "benchmark" problem we will run into. Similarly, looking at a data set alone, one cannot produce any reliable estimate for the noise variance. Perhaps we "feel" as though we ought to be able to (for how often can it be heard: "This data set is clearly noisy"), but only because we already have a smoothness prior built into our neural network. Why? Because the types of functions we tend to encounter in practice are smooth.

NFL focuses our attention on the importance of the prior information available. One should not attempt to find learning systems that are universally good. Instead, one should study the possibilities that various priors present. Unfortunately, identifying and justifying the prior for the problem at hand is already a daunting task, let alone incorporating it into the learning problem. One way would be through the use of hints (Abu-Mostafa, 1995; Sill, 1998; Sill \& Abu-Mostafa, 1997). A possible starting point would be to identify priors, $P[f]$, with learning systems that perform well with respect to them (for example, Barber \& Williams, 1997; Zhu \& Rohwer, 1996). One might then be able to relate real problems to these priors and thus choose an appropriate learning system, or at least it might become possible to make precise the assumptions behind a belief that a certain learning system is appropriate for a certain problem. A useful result would be of the form, "Algorithm A [or learning system A] performs better than average on problems drawn from the (useful) class B."

\section{Appendix}

A.1 Estimating Noise Variance Using Linear Models. We have $\hat{A}$, an estimate of $\sigma^{2}(d+1)+B$, and we would like to estimate $B$ by bootstrap-

\footnotetext{
${ }^{4}$ VC results make statements about $\operatorname{Pr}$ [training error|test error] by making the statement that the probability of a low training error given a high test error is extremely unlikely. In practice, what we would really like to make statements about is the $\operatorname{Pr}[$ test error|training error] for which we really need to make some statement about the a priori probablity of a certain test error; that is, we need to make some statement about a prior over target functions.
} 
ping. Suppose we draw $N_{B}$ data points and estimate $B$ (using the bootstrap technique) by $\hat{B}$. It can be shown that (see theorem 5 )

$$
\langle\hat{B}\rangle_{\mathbf{n}}=\tilde{B}+\sigma^{2}(d+1)\left(1-\frac{N_{B}}{N}\right)+\frac{\sigma^{2}}{N} \underbrace{\operatorname{tr}\left\langle\Sigma^{-1} \mathbf{V} \Sigma^{-1} \mathbf{V}\right\rangle}_{O(d+1)},
$$

where $\tilde{B}$ is an unbiased estimate of $B$ and the expectation in the last term is with respect to the bootstrapping. The definitions of $\mathbf{V}$ and $\Sigma$ are given in the next section and are unimportant for our purpose here. Thus, we now have two quantities: $\hat{A}$, an estimate of $B+\sigma^{2}(d+1)$, and $\hat{B}$, an estimate of $B+\sigma^{2}\left((d+1)\left(1-N_{B} / N\right)+\operatorname{tr}\left\langle\Sigma^{-1} \mathbf{V} \Sigma^{-1} \mathbf{V}\right\rangle / N\right)$. Solving for $\sigma^{2}$, we have

$$
\sigma^{2}=\frac{\hat{A}-\hat{B}}{(d+1) \frac{N_{B}}{N}-\frac{\operatorname{tr}\left\langle\Sigma^{-1} \mathbf{V} \Sigma^{-1} \mathbf{V}\right\rangle}{N}} .
$$

However, we are bootstrapping a quantity $B$, which is related to the deviation of a statistical quantity of the sample from its population value. This estimate is good only as long as $N$ represents the population-that is, $N_{B} / N \rightarrow 0$. In this case, $\hat{B}=B+\sigma^{2}(d+1)$ and thus we cannot combine this with $\hat{A}$ to solve for $\sigma^{2}$ (the expression in equation A.2 becomes indeterminate). We are faced with a dilemma. For finite $N_{B} / N$ we can get an estimate of $\sigma^{2}$, but this estimate is not very good. However, we have no way to make it better because by making the bootstrap more effective for $B$, the final result cannot be solved for $\sigma^{2}$. Technically speaking, we cannot in this way get a consistent estimate of $\sigma^{2}$, even as $N \rightarrow \infty$.

A.2 Proof of Theorems. We use the notation

$$
\begin{aligned}
& \mathbf{X}=\left[\begin{array}{llll}
\mathbf{x}_{1} & \mathbf{x}_{2} & \ldots & \mathbf{x}_{N}
\end{array}\right], \quad \mathbf{y}=\mathbf{f}+\mathbf{n} \\
& \Sigma \equiv\left\langle\mathbf{x x}^{T}\right\rangle_{\mathbf{x}}, \quad \mathbf{q}=\langle\mathbf{x} f(\mathbf{x})\rangle_{\mathbf{x}} \text {. }
\end{aligned}
$$

$\mathbf{X}$ is a $(d+1) \times N$ matrix where the $\mathbf{x}_{i}$ are the data points (we use the convention that the first component is set to 1 and the remaining $d$ components are the coordinates of the data point). $\mathbf{f}$ is an $N \times 1$ vector of output values at the data points, and $\mathbf{n}$ is a vector of noise realizations. If $\langle\mathbf{x}\rangle=0$, then $\Sigma$ is the covariance matrix for the input distribution. The law of large numbers gives us that $\mathbf{X} \mathbf{X}^{T} / N \underset{N \rightarrow \infty}{\longrightarrow} \Sigma$ and $\mathbf{X f} / N \underset{N \rightarrow \infty}{\longrightarrow} \mathbf{q}$, where we assume that the conditions for this to happen are satisfied. We thus define $\mathbf{V}$ and $\mathbf{a}$ by

$$
\frac{\mathbf{X} \mathbf{X}^{T}}{N}=\Sigma+\frac{\mathbf{V}(\mathbf{X})}{\sqrt{N}}, \quad \frac{\mathbf{X} \mathbf{f}}{N}=\mathbf{q}+\frac{\mathbf{a}(\mathbf{X})}{\sqrt{N}} .
$$

Note that $\langle\mathbf{V}\rangle_{\mathbf{X}}=\langle\mathbf{a}\rangle_{\mathbf{X}}=0$ and $\operatorname{Var}(\mathbf{V})$ and $\operatorname{Var}(\mathbf{a})$ are $O(1)$. 
Theorem 4. Let the learning model be the set of linear functions $\mathbf{w} \cdot \mathbf{x}$ and let the learning algorithm be minimization of the squared error. Then

$$
\left\langle\mathcal{E}_{t r}^{*}\right\rangle_{\mathcal{D}, \mathbf{n}}=E_{0}+\sigma^{2}-\frac{\sigma^{2}(d+1)+B}{N}+O\left(\frac{1}{N^{\frac{3}{2}}}\right),
$$

where $E_{0}$ and $B$ are constants given by

$$
\begin{aligned}
& E_{0}=\left\langle f^{2}\right\rangle-\mathbf{q}^{T} \Sigma^{-1} \mathbf{q} \\
& B=\frac{\left\langle\mathbf{q}^{T} \Sigma^{-1} \mathbf{V} \Sigma^{-1} \mathbf{V} \Sigma^{-1} \mathbf{q}+\mathbf{a}^{T} \Sigma^{-1} \mathbf{a}-2 \mathbf{a}^{T} \Sigma^{-1} \mathbf{V} \Sigma^{-1} \mathbf{q}\right\rangle}{N} .
\end{aligned}
$$

Proof. The least squares estimate of $\mathbf{w}$ is given by

$$
\hat{\mathbf{w}}=\left(\mathbf{X} \mathbf{X}^{T}\right)^{-1} \mathbf{X} \mathbf{y}
$$

from which we calculate the expected training error as

$$
\begin{aligned}
\left\langle\mathcal{E}_{t r}\right\rangle & =\left\langle\frac{1}{N}\left(\mathbf{X}^{T} \hat{\mathbf{w}}-\mathbf{y}\right)^{2}\right\rangle=\left\langle\frac{1}{N}\left(\left(\mathbf{X}^{T}\left(\mathbf{X} \mathbf{X}^{T}\right)^{-1} \mathbf{X}-\mathbf{1}\right) \mathbf{y}\right)^{2}\right\rangle \\
& =\frac{\left\langle\mathbf{f}^{T} \mathbf{f}\right\rangle-\left\langle f^{T} \mathbf{X}\left(\mathbf{X} \mathbf{X}^{T}\right)^{-1} \mathbf{X} \mathbf{f}\right\rangle+\left\langle\mathbf{n}^{T} \mathbf{n}\right\rangle-\left\langle\mathbf{n}^{T} \mathbf{X}^{T}\left(\mathbf{X} \mathbf{X}^{T}\right)^{-1} \mathbf{X} \mathbf{n}\right\rangle}{N} \\
& =\underbrace{\left\langle f^{2}\right\rangle-\frac{\left\langle\mathbf{f}^{T} \mathbf{X}^{T}\left(\mathbf{X} \mathbf{X}^{T}\right)^{-1} \mathbf{X} \mathbf{f}\right\rangle}{N}}_{T_{1}}+\sigma^{2}-\frac{\sigma^{2}(d+1)}{N} .
\end{aligned}
$$

Using equation A.3 and the identity $[1+\lambda \mathbf{A}]^{-1}=1-\lambda \mathbf{A}+\lambda^{2} \mathbf{A}^{2}+O\left(\lambda^{3}\right)$, we evaluate $T_{1}$ as

$$
\begin{aligned}
T_{1}= & \left\langle f^{2}\right\rangle-\mathbf{q}^{T} \Sigma^{-1} \mathbf{q} \\
& -\frac{\left\langle\mathbf{q}^{T} \Sigma^{-1} \mathbf{V} \Sigma^{-1} \mathbf{V} \Sigma^{-1} \mathbf{q}+\mathbf{a}^{T} \Sigma^{-1} \mathbf{a}-2 \mathbf{a}^{T} \Sigma^{-1} \mathbf{V} \Sigma^{-1} \mathbf{q}\right\rangle}{N}+O\left(\frac{1}{N^{\frac{3}{2}}}\right) .
\end{aligned}
$$

The theorem now follows.

This result is similar to results obtained by Amari, Murata, Müller, and Yang (1997) and Moody (1991). From theorem 4, we have that

$$
B=\frac{1}{N}(\underbrace{\left\langle\mathbf{q}^{T} \Sigma^{-1} \mathbf{V} \Sigma^{-1} \mathbf{V} \Sigma^{-1} \mathbf{q}\right\rangle}_{T_{1}}+\underbrace{\left\langle\mathbf{a}^{T} \Sigma^{-1} \mathbf{a}\right\rangle}_{T_{2}}-2 \underbrace{\left\langle\mathbf{a}^{T} \Sigma^{-1} \mathbf{V} \Sigma^{-1} \mathbf{q}\right\rangle}_{T_{3}}) .
$$


We wish to estimate $T_{1}, T_{2}, T_{3}$. Suppose that we try to bootstrap them as follows. For $T_{1}$, we take $\hat{\mathbf{q}}=\mathbf{X y} / N=\mathbf{X}(\mathbf{f}+\mathbf{n}) / N$ and $\hat{\Sigma}=\left(\mathbf{X X}^{T} / N\right)$. We estimate $\mathbf{V}$ by sampling $N_{B}$ of the $N$ data points and compute $\sqrt{N_{B}}\left(\left(\mathbf{X}_{B} \mathbf{X}_{B}^{T} / N_{B}\right)-\right.$ $\left.\left(\mathbf{X X}^{T} / N\right)\right)$. We then estimate $T_{1}$ by $\hat{T}_{1}$, a bootstrapped expectation of $\hat{\mathbf{q}}^{T} \hat{\Sigma}^{-1} \mathbf{V} \hat{\Sigma}^{-1} \mathbf{V} \Sigma^{-1} \hat{\mathbf{q}}$. This requires $N_{B} / N$ to be small. Doing all this, we find that

$$
\hat{T}_{1}=\frac{\mathbf{f}^{T} \mathbf{X}^{T}}{N}\left\langle\hat{\Sigma^{-1}} \mathbf{V} \hat{\Sigma}^{-1} \mathbf{V} \hat{\Sigma}^{-1}\right\rangle \frac{\mathbf{X f}}{N}+\frac{\sigma^{2}}{N} \operatorname{tr}\left\langle\hat{\Sigma^{-1}} \mathbf{V} \hat{\Sigma}^{-1} \mathbf{V}\right\rangle,
$$

where we have an expectation with respect to the noise and the remaining expectation is with respect to the bootstrapping. Notice that the first term is the unbiased estimator of $T_{1}$. Denote this term by $\tilde{T}_{1}$.

For $\hat{T}_{2}=\left\langle\mathbf{a}^{T} \Sigma^{-1} \mathbf{a}\right\rangle$ we use $\mathbf{a}=\sqrt{N_{B}}\left(\mathbf{X}_{B} \mathbf{y}_{B} / N_{B}-\hat{\mathbf{q}}\right)$. Partitioning the input matrix and noise vector into the part in the sampled $N_{B}$ points and the remaining part, write $\mathbf{X}=\left[\mathbf{X}_{B} \tilde{\mathbf{X}}_{B}\right]$ and $\mathbf{n}=\left[\mathbf{n}_{B}^{T} \tilde{\mathbf{n}}_{B}^{T}\right]^{T}$. Noting that $\mathbf{n}_{B}$ and $\tilde{\mathbf{n}}_{B}$ are independent, we have for $\hat{T}_{2}$ :

$$
\begin{aligned}
\hat{T}_{2} & =\tilde{T}_{2}+\frac{1}{N^{2} N_{B}}\left(N_{B} \mathbf{X} \mathbf{n}-N \mathbf{X}_{B} \mathbf{n}_{B}\right) \Sigma^{-1}\left(N_{B} \mathbf{X} \mathbf{n}-N \mathbf{X}_{B} \mathbf{n}_{B}\right) \\
& =\tilde{T}_{2}+\frac{N_{B}^{2}\left(N-N_{B}\right)^{2}}{N^{2} N_{B}}\left[\frac{\tilde{\mathbf{X}}_{B} \tilde{\mathbf{n}}_{B}}{N-N_{B}}-\frac{\tilde{\mathbf{X}}_{B} \mathbf{n}_{B}}{N_{B}}\right] \Sigma^{-1}\left[\frac{\tilde{\mathbf{X}}_{B} \tilde{\mathbf{n}}_{B}}{N-N_{B}}-\frac{\tilde{\mathbf{X}}_{B} \mathbf{n}_{B}}{N_{B}}\right] \\
& =\tilde{T}_{2}+\sigma^{2}(d+1)\left(1-\frac{N_{B}}{N}\right)+o\left(\frac{1}{N}\right),
\end{aligned}
$$

where we have taken the expectation with respect to the noise and $\tilde{T}_{2}$ is an unbiased estimate of $T_{2}$.

Performing the same kind of analysis for $\hat{T}_{3}$, we find that $\hat{T}_{3}=\tilde{T}_{3}$, an unbiased estimate of $T_{3}$. Now we estimate $\hat{B}$ by $\hat{T}_{1}+\hat{T}_{2}-2 \hat{T}_{3}$, which is the unbiased estimate of $B$ plus some correction terms. Therefore we have proved the following theorem.

Theorem 5. For $N \rightarrow \infty, N_{B} / N \rightarrow 0$, the bootstrapped estimate of $B$, which we call $\hat{B}$, has an expectation given by

$$
\langle\hat{B}\rangle_{\mathbf{n}}=\tilde{B}+\frac{\sigma^{2}(d+1)}{N}\left(1-\frac{N_{B}}{N}\right)+\frac{\sigma^{2}}{N} \operatorname{tr}\left\langle\Sigma^{-1} \mathbf{V} \Sigma^{-1} \mathbf{V}\right\rangle_{\mathbf{X}},
$$

where $\tilde{B}$ is an unbiased estimate of $B$ and the remaining expectation is with respect to the bootstrapping. 


\section{Acknowledgments}

We thank Yaser Abu-Mostafa and the members of the Caltech Learning Systems Group for helpful comments. In addition, two anonymous referees provided useful comments.

\section{References}

Abu-Mostafa, Y. (1995). Hints. Neural Computation, 4(7), 639-671.

Amari, S., Murata, N., Müller, K., \& Yang, H. (1997). Asymptotic statistical theory of overtraining and cross validation. IEEE Transactions on Neural Networks, $8(5), 985-996$.

Barber, D., \& Williams, C. K. I. (1997). Gaussian processes for Bayesian classifications via hybrid Monte Carlo. In M. C. Mozer, M. I. Jordan, \& T. Petsche (Eds.), Advances in neural information processing systems, 9 (pp. 340-346). San Mateo, CA: Morgan Kaufmann.

Cataltepe, Z., Abu-Mostafa, Y. S., \& Magdon-Ismail, M. (1999). No free lunch for early stopping. Neural Computation, 11, 995-1001.

Cortes, C., Jackel, L. D., \& Chiang, W.-P. (1994). Limits on learning machine accuracy imposed by data quality. In G. Tesauro, D. S. Touretzky, \& T. K. Leen (Eds.), Neural information processing systems, 7 (pp. 239-246). Cambridge, MA: MIT Press.

Cover, T. M., \& Thomas, J. A. (1991). Elements of information theory. New York: Wiley.

Moody, J. E. (1991). The effective number of parameters: An analysis of generalization and regularization in nonlinear learning systems. In J. E. Moody, S. J. Hanson, \& R. P. Lippmann (Eds.), Advances in neural information procesing systems, 4 (pp. 847-854). San Mateo, CA: Morgan Kaufmann.

Shao, J., \& Tu, D. (1996). The jackknife and the bootstrap. New York: Springer-Verlag.

Sill, J. (1998). Monotonic networks. In M. Kearns, M. Jordan, \& S. Solla (Eds.), Advances in neural information processing systems, 10. Cambridge, MA: MIT Press.

Sill, J., \& Abu-Mostafa, Y. S. (1997). Monotonicity hints. In M. C. Mozer, M. I. Jordan, \& T. Petsche (Eds.), Advances in neural information processing systems, 9 (pp. 634-640). Cambridge, MA: MIT Press.

Vapnik, V. N. (1995). The nature of statistical learning theory. New York: SpringerVerlag.

Wolpert, D. H. (1995). The mathematics of generalization. Reading, MA: Addison Wesley.

Wolpert, D. H. (1996a). The existence of a priori distinctions between learning algorithms. Neural Computation, 8(7), 1391-1420.

Wolpert, D. H. (1996b). The lack of a priori distinctions between learning algorithms. Neural Computation, 8(7), 1341-1390.

Wolpert, D. H., \& Macready, W. G. (1997). No free lunch theorems for optimization. IEEE Transactions on Evolutionary Computation, 1, 67-82. 
Zhu, H. (1996). No free lunch for cross validation. Neural Computation, 8(7), 1421-1426.

Zhu, H., \& Rohwer, R. (1996). Bayesian regression filters and the issue of priors. Neural Computation Applications, 4, 130-142.

Received May 29, 1998; accepted March 31, 1999. 